\title{
Factors Affecting the Incidence of Wound Infection in Patients after Valve Replacement
}

\author{
Authors \\ Mohsen Mohamed Abdelkrem', Yasser Shaban Mubarak², Faisal Amr Mourad', \\ Mostafa Mohamed Elsayed ${ }^{4}$ \\ ${ }^{1}$ Department of Cardiothoracic Surgery, Faculty of Medicine, Ain-shams University, Egypt \\ Email:mohsen@yahoo.com \\ ${ }^{2}$ Department of Cardiothoracic Surgery, Faculty of Medicine, Minia University, Egypt \\ Email: yasser@yahoo.com \\ ${ }^{3}$ Department of Cardiothoracic Surgery, Faculty of Medicine, Ain-shams University, Egypt \\ Email:faisal@hotmail.com \\ ${ }^{4}$ Department of Cardiothoracic Surgery, Faculty of Medicine, Minia University, Egypt \\ Email:mostafa@yahoo.com
}

\begin{abstract}
Introduction: Postoperative sternal wound infections cause considerable extra morbidity, mortality and costs. This study was conducted in the Unit of Cardiothoracic Surgery, Minia University Hospital, Department of Cardiothoracic Surgery, Ain-shams University Hospital undergoing valve replacement in the period between July 2016 to July 2017 on 108 patients undergoing valve replacement. Included both gender from 16 to 70 years elective cases after exclusion association with CABG patients.

Subject and Methods: We selected 108 patients undergoing valve replacement. We performed a one-year centre analysis of prospective surveillance in patients having cardiac surgery. The patients' charts were reviewed and data were entered into a computer spread sheet.

Results: This prospective study included 108 consecutive patients who underwent cardiac surgery for heart valve disease during the study period. Age of all patients ranged from 18 to 65 years with mean \pm standard deviation of $38.07 \pm 12.35$ years.

Conclusion: Despite many advances in prevention, sternal wound infection remains a significant postoperative complication after cardiac surgery.

Keywords: Incidence, Wound Infection, Valve.
\end{abstract}

\section{Introduction}

ranges from $1 \%$ to $5 \%$ (Gummert et al., 2002). The

Despite optimized surgical technique and perioperative antibiotic prophylaxis, the incidence of sternal wound infection after cardiac surgery mortality for patients with deep sternal wound infection (DSWI) may be twice as high as those without. The development of sternal infection is 
associated not only with increased morbidity and death but also with prolonged hospital stay and higher costs (Braxton et al., 2008). Sternal wound infection is a devastating and fatal complication. They can be divided into superficial infections and deep infections, based on the depth of the infection in the wound. Early infections include both superficial infections, reaching the dermis and subcutaneous tissue, and deep sternal wound infections (DSWIs) that reach under the sternum and the anterior mediastinum. DSWI is also referred to as postoperative mediastinitis, which can present either early or as a late infection. Late infections include osteomyelitis, subcutaneous abscess, and sternocutaneous fistulas (SCFs) (Horan et al., 2008). According to Centers for Disease Control and Prevention (CDC) guidelines, the definition of a DSWI requires positive culture results of surgical sites or drainage from the mediastinal area or evidence of infection during surgical re-exploration or fever, sternal instability, and positive blood culture results (Mangram et al., 1999). Multiple modifiable and non-modifiable risk factors have been associated with DSWI. Some of these factors are related to patient characteristics, whereas others are related to operative or perioperative management (Fowler et al., 2005). Patient factors include age, sex, obesity, diabetes mellitus or hyperglycemia during the perioperative period, smoking, staphylococcus aureus nasal carriage, skin infection anywhere on the body, chronic obstructive pulmonary disease, heart failure, kidney dysfunction, peripheral vascular disease, and emergent or urgent surgery (Cayci et al., 2008). Surgical risk factors include prolonged aortic crossclamp time, cardiopulmonary bypass time or total surgical time, inadvertent Para median sternotomy, use of bone wax, extensive use of electro cauterization, procedures requiring prosthesis implant, use of intra-aortic balloon pump, postoperative bleeding, blood transfusions, reexploration for bleeding, redo operation, prolonged mechanical ventilation, and prolonged ICU stay (Fakih et al., 2007). Strategies of treatment include surgical revision with primary closure, surgical revision with open dressing or closed irrigation, reconstruction with soft tissue flap, and application of vacuum (Steingrimsson et al., 2012).

\section{Subjects and Methods}

This study was conducted in the Unit of Cardiothoracic Surgery, Minia University Hospital, Department of Cardiothoracic Surgery, Ain-shams University Hospital in patients undergoing valve replacement in the period between July 2016 to July 2017.

We selected 108 patients undergoing valve replacement. We performed a one-year centre analysis of prospective surveillance in patients having cardiac surgery. The patients' charts were reviewed and data were entered into a computer spread sheet.

Because this study involved human subjects, approval was sought and obtained from the faculty's ethical committee in each participating hospital. Informed consent had been obtained from each patient to allow his or her data to be entered into the research.

\section{Inclusion criteria}

- Age from 16 years old till 70 years old.

- Male and female gender

- Elective open single and double valve replacement and repair (with or without) tricuspid valve repair.

- Redo open valve replacement

\section{Exclusion criteria}

- Age< 16 years.

- Emergency Cases needing for valve replacement.

- Open valve replacement associated with CABG

- Diagnostic criteria:

- Time of appearance of infection within one month from operation.

- Antibiotics preoperative for all patients

- Superficial sternal wound infection include skin and subcutaneous till muscle layer.

- Deep sternal wound infection: include sternal and underlying mediastinum. 
All patients underwent the following evaluations:

Preoperatively:

History and physical examination:

Age, gender, history of smoking, hypertension, diabetes mellitus (type1 and 2), history of cerebrovascular accident (CVA), COPD, peripheral arterial disease, coronary artery disease, redo heart surgery, NYHA function class, renal impairment, any previous operation, allergy or drug intake, ENT and dermatological examination for exclusion of septic focus.

Investigations:

Radiological

Echocardiograph: detection of valve lesion and indication of valve replacement, $\mathrm{EF}$, left atrial enlargement, chamber dimension of the heart.

Coronary angiography for patient above 40 years.

Carotid duplex for atherosclerotic patients.

Chest x-ray

ECG

Laboratory:

Complete blood count

Blood sugar.

Glyclated HB (HBA1C)

Liver function tests.

Renal function tests.

Lipid profile (cholesterol, triglycerides, LDL, HDL).

Prothrombin time, concentration and INR.

Preoperative:

Measuring weight and height then calculating body mass index, body surface area and full flow.

Infection control strategies as method of hair removal by shaving or clipping, location of removal operation room or surgical ward, time of removal, who removed the hair either by nurse or by patient, surgeon. Ways of skin preparation betadine paint VS alcohol and iodine. Frequency of shower day or day and night and the solution was used antiseptic soap.

Antibiotic preoperative for all patients and the time of administration one hour before surgery.

Recording operation time (skin to skin), by pass time, cross-clamp time, hemostasis was done usage of diathermy and bone wax, units of blood transfusion.

Midline sternotomy by sternal saw then open pericardium and cannulation of aorta, vena cava and cardioplegia cannula, insertion of aortic cross clamp after that replace the diseased valve with certain size mechanical or tissue valve according to annulus of the valve, then closure of aortotmy in case of aortic valve replacement or atriotomy in other valves. Then removal of cannulations and good hemostasis. Eventually anatomical closure of sternum either by simple wire or figure of eight with drains.

Postoperatively:

ICU monitoring:

Number of blood or plasma transfusions $(<48 \mathrm{~h})$

Amount of blood loss in ICU

Resternotomy for bleeding either in ICU or OR

If patient is on inotropes support or not

Mechanical ventilation time

Length of stay (LOS): ICU duration stay and total hospital stay

Full lab and follow up RBS in diabetic patient.

In ward monitoring

Dressing by betadine and topical antibiotic spray.

Repeated dressing if wound infected and swab for culture, sensitivity.

Superficial wound infection and treated by repeated dressing and good coverage antibiotic in addition correction of general condition as hemoglobin, nutritional status assessment, albumin, blood glucose level, physiotherapy, monitoring anticoagulant drug and control of sepsis. 


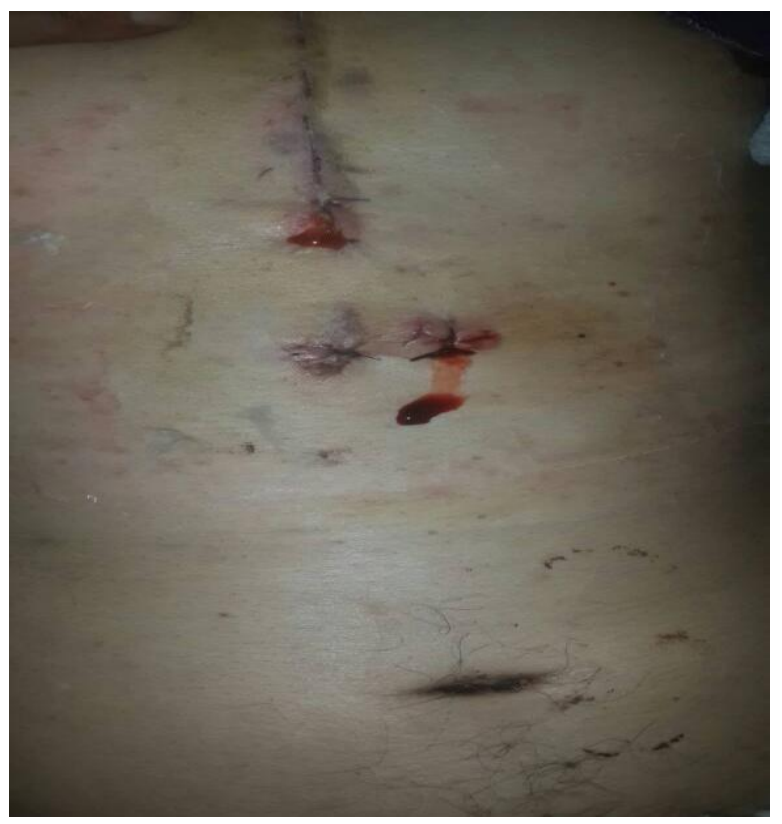

Fig. (1): Male patient 65 years after DVR with superficial sternal wound infection before treatment.

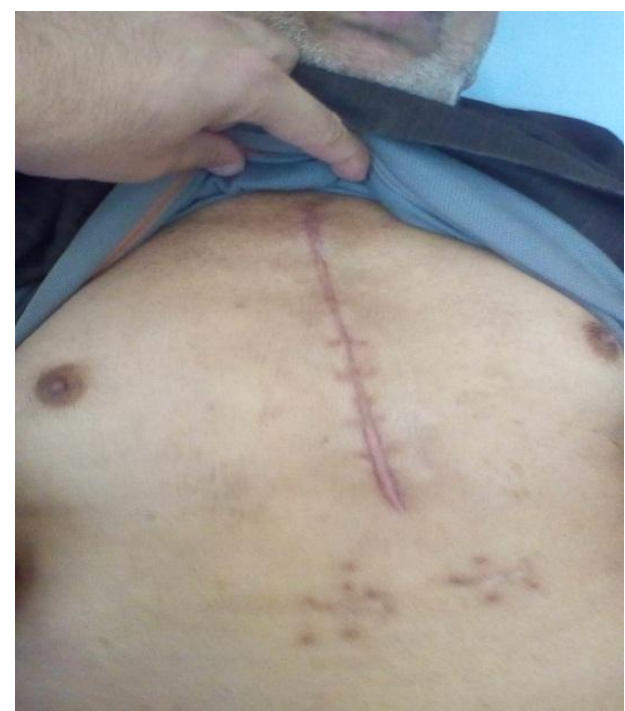

Fig. (2): male patient 65 years after DVR with superficial sternal wound infection after treatment. In deep wound infection was managed by the previous steps, in addition, one of the cases, drained by vacuum then surgical debridement and the other two patient debridement from the start after repeated dressing by squeezing wound.

In addition, the following information was collected for patients presented with DSWI or SSWI:

Type of infection, time from primary operation to infection, antibiotic treatment, surgical treatment, outcome.

Follow up patient after discharging and readmission cases of wound infection if discovered later about period of one month.

Blood cultures: for patient with deep surgical wound infection.

Bacterial cultures: swab from infected wound.

Chest enhanced computed tomography (CT): for deep wound infection patient showing retrosternal collection or pleural effusion.

TEE for exclusion of vegetation infective endocarditis

Follow up fever chart, blood glucose level, number of soaked dressing and drains or radivac if present.

Follow up chest $x$ ray and laboratory investigation as HB level, TLC, INR, platelet, urea and creatnine, liver function.

Procedures for treatment DSWI:

Wounds manifest with purulent drainage and loose wire. Vacuum-assisted closure (VAC) is performed for wound preparation, or wound dressing is used for Open wounds, lavage using antibiotic saline, removal of old sternal wires, and excision of all wound edges including skin, subcutaneous tissue, and necrotic tissue in the mediastinum. To achieve complete wound closure in the pericardium and under the sternum, drains are placed after rewiring the sternal wire and performing subcutaneous drainage.

Figure $(2,3)$ male patient 55 years after DVR with mediastinitis.
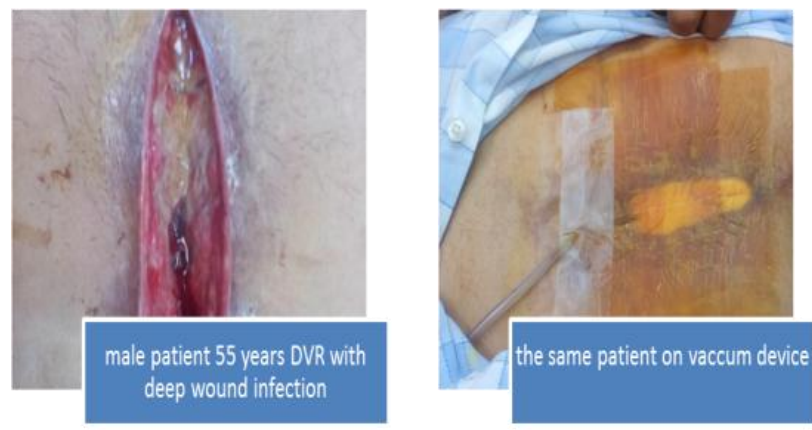

Fig. (3): Male patient 55 years after DVR with mediastinitis.

Fig. (4): Male patient 55 years after after ten days of NPWT.

The same patient as in previous Figures after ten days of NPWT. The wound is clean and the wound edges are well vascularized. 


\section{Statistical Analysis}

Data were collected, revised, coded and entered to the Statistical Package for Social Science (IBM SPSS) version 20. The qualitative data were presented as number and percentages.

The comparison between two independent groups with qualitative data was done by using Chi-square test.

Logistic regression analysis was used to assess the significant predictors of risk factors wound infection.

The confidence interval was set to $95 \%$ and the margin of error accepted was set to $5 \%$. So, the pvalue was considered significant as the following:

$$
\begin{aligned}
& P>0.05 \text { : Non significant } \\
& P<0.05 \text { : Significant } \\
& P<0.01 \text { : Highly significant. }
\end{aligned}
$$

\section{Results}

This prospective study included 108 consecutive patients who underwent cardiac surgery for heart valve disease during the study period. Age of all patients ranged from 18 to 65 years with mean \pm standard deviation of $38.07 \pm 12.35$ years. Table (1) represents preoperative demographic characteristics of all studied patients. One hundred patients $(92.59 \%)$ were $<60$ years old and 8 patients $(7.40 \%)$ were $\geq 60$ years old. Sixty patients $(55.55 \%)$ were female and $48(44.44 \%)$ were male. Seventy two patients $(66.66 \%)$ were non-obese (BMI $<30 \mathrm{~kg} / \mathrm{m} 2$ ), and the remaining 38 patients $(35.2 \%)$ were obese (BMI $\geq 30 \mathrm{~kg} / \mathrm{m} 2)$.

Table (1): Preoperative demographic characteristics of all studied patients

\begin{tabular}{|c|c|c|}
\hline Variables & $\begin{array}{c}\text { Numbe } \\
\mathrm{r}(\mathrm{n}=108)\end{array}$ & Percent \\
\hline Age: & & \\
\hline$<60$ years & 100 & $92.59 \%$ \\
\hline$\geq 60$ years & 8 & $7.40 \%$ \\
\hline Gender: & & \\
\hline Male & 48 & $44.44 \%$ \\
\hline Female & 60 & $55.55 \%$ \\
\hline Obesity: & & \\
\hline Non-obese & 72 & $66.66 \%$ \\
\hline Obese & 38 & $35.2 \%$ \\
\hline
\end{tabular}

Table (2) represents preoperative clinical risk factors in all studied patients. These preoperative clinical risk factors were: current smoking (35.18\%), anemia (37.03\%), diabetes (25.92\%), elevated HbAlc (20.37\%), hypertension (24.07\%), peripheral vascular disease $(5.55 \%)$, cerebrovascular disease (12.96\%), renal dialysis $(7.40 \%)$, chronic pulmonary disease $(14.81 \%)$, hepatitis C $(25.92 \%)$, and previous cardiac surgery $(11.11 \%)$.

Table (2): Preoperative clinical risk factors in all studied patients

\begin{tabular}{|c|c|c|}
\hline Variables & Number $(\mathrm{n}=108)$ & Percent \\
\hline Current smoking & 38 & $35.18 \%$ \\
\hline Anemia & 40 & $37.03 \%$ \\
\hline Diabetes mellitus & 28 & $25.92 \%$ \\
\hline Elevated HA1c > 7\% & 22 & $20.37 \%$ \\
\hline Hypertension & 26 & $24.07 \%$ \\
\hline Peripheral vascular disease & 6 & $5.55 \%$ \\
\hline Cerebrovascular disease & 14 & $12.96 \%$ \\
\hline Renal dialysis & 8 & $7.40 \%$ \\
\hline Chronic pulmonary disease & 16 & $14.81 \%$ \\
\hline Hepatitis C & 28 & $52.92 \%$ \\
\hline Previous cardiac surgery & 12 & $11.11 \%$ \\
\hline
\end{tabular}

Table (3) represents preoperative echocardiographic results of all studied patients. The important echocardiographic findings were: low ejection frcation $(33.33 \%)$, dilated left atrium $(74.07 \%)$, severe pulmonary hypertension (50\%), mitral valve disease $(77.77 \%)$, aortic valve disease $(51.85 \%)$ and moderate to severe tricuspid valve regurgitation $(57.40 \%)$.

Table (3): Preoperative echocardiographic results of all studied patients

\begin{tabular}{|l|c|c|}
\hline Variables & $\begin{array}{c}\text { Number } \\
(\mathrm{n}=108)\end{array}$ & Percent \\
\hline Low ejection fraction & 36 & $33.33 \%$ \\
\hline Dilated left atrium & 80 & $74.07 \%$ \\
\hline Severe pulmonary hypertension & 54 & $50 \%$ \\
\hline Mitral valve disease & 84 & $77.77 \%$ \\
\hline Aortic valve disease & 56 & $51.85 \%$ \\
\hline $\begin{array}{l}\text { Moderate/severe tricuspid } \\
\text { regurge }\end{array}$ & 62 & $57.40 \%$ \\
\hline
\end{tabular}

Table (4) represents preoperative anti-septic measures in all studied patients. These measures were: hair removal by shaving $(85.18 \%)$ or clipping (14.81\%), skin preparation by Alcohol only $(5.55 \%)$, Betadine only $(66.66 \%)$, on combination 
$(27.77 \%)$, shower at night $(48.14 \%)$, or at day and night $(51.85 \%)$, and use of showering antiseptic $(50 \%)$.

Table (4): Preoperative anti-septic measures in all studied patients

\begin{tabular}{|l|c|c|}
\hline Variables & Number $(\mathrm{n}=108)$ & Percent \\
\hline Hair removal: & & \\
\hline Shaving & 92 & $85.18 \%$ \\
\hline Clipping & 16 & $14.81 \%$ \\
\hline Skin preparation: & & \\
\hline Alcohol & 6 & $5.55 \%$ \\
\hline Betadine & 72 & $66.66 \%$ \\
\hline Betadine and Alcohol & 30 & $27.77 \%$ \\
\hline Shower: & & \\
\hline Night & 52 & $48.14 \%$ \\
\hline Day and night & 56 & $51.85 \%$ \\
\hline Showering antiseptic: & & \\
\hline No & 54 & $50 \%$ \\
\hline Yes & 54 & $50 \%$ \\
\hline
\end{tabular}

Table (5) represents operative and postoperative outcomes in all studied patients. The unfavorable outcomes of interest were: prolonged bypass time > $120 \mathrm{~min}(11.11 \%)$, prolonged cross clamp time $>60$ $\min (51.85 \%)$, blood loss $>500 \mathrm{ml}$ in ICU $(22.22 \%)$, re-exploration for bleeding (5.55\%), prolonged ventilation time $>12$ hours $(9.25 \%)$, removal of drains after 3 days $(59.25 \%)$, and sternal wound infection $(22.22 \%)$.

Table (5): Operative and postoperative outcomes in all studied patients

\begin{tabular}{|l|l|l|}
\hline Variables & Number $(\mathrm{n}=108)$ & Percent \\
\hline Bypass time $>120 \mathrm{~min}$ & 12 & $11.11 \%$ \\
\hline Cross clamp time $>60 \mathrm{~min}$ & 56 & $51.85 \%$ \\
\hline Blood loss $>500 \mathrm{ml}$ & 24 & $22.22 \%$ \\
\hline Re-exploration for bleeding & 6 & $5.55 \%$ \\
\hline Ventilation time $>12 \mathrm{~h}$ & 10 & $9.25 \%$ \\
\hline Removal of drains after 3 days & 64 & $59.25 \%$ \\
\hline Sternal wound infection & 24 & $22.22 \%$ \\
\hline
\end{tabular}

Table (6) and Figure (16) represent incidences of different types of sternal wound infection. There were no sternal wound infection in 87 patients (77.77\%), superficial sternal wound infection in 21 patients $(19.44 \%)$ and deep sternal wound infection in 3 patients $(2.77 \%)$.
Table (6): Incidences of different types of sternal wound infection

\begin{tabular}{|l|c|c|}
\hline Variables & $\begin{array}{c}\text { Number } \\
(\mathrm{n}=108)\end{array}$ & Percent \\
\hline No sternal wound infection & 84 & $77.77 \%$ \\
\hline Superficial sternal wound infection & 21 & $19.44 \%$ \\
\hline Deep sternal wound infection & 3 & $2.77 \%$ \\
\hline
\end{tabular}

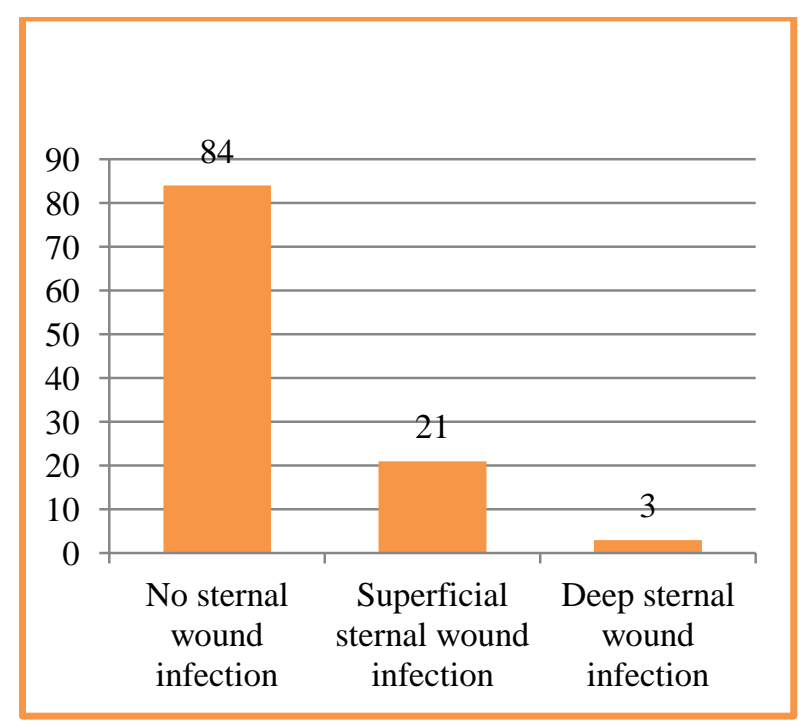

Fig. (5): Incidences of different types of sternal wound infection.

Table (7) and Figure (6) represent clinical presentation of 24 patients with sternal wound infections. Sixteen patients $(67 \%)$ had wound discharge, and 8 patients $(33 \%)$ had wound discharge and fever.

Table (7): Clinical presentation of 24 patients with sternal wound infections

\begin{tabular}{|l|c|c|}
\hline Variables & Number $(\mathrm{n}=24)$ & Percent \\
\hline Wound discharge & 16 & $67 \%$ \\
\hline Wound discharge and fever & 8 & $33 \%$ \\
\hline
\end{tabular}

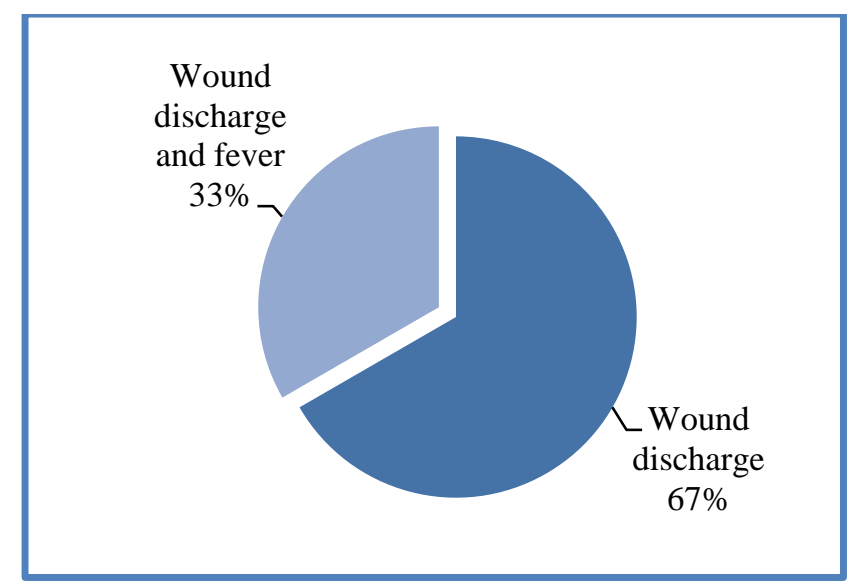

Fig. (6): Clinical presentation of 24 patients with sternal wound infections. 
Table (8) and Figure (7) represent microbiological results in 24 patients with sternal wound infection. The causative organisms were staph. aureus in $33.33 \%$, staph. epidermis in $16.66 \%$, staph pyogenes in $25 \%$, streptococcus epidermis in $4.16 \%$ and E-coli in $12.5 \%$. There was no growth in $8.33 \%$.

Table (8): Microbiological results in 24 patients with sternal wound infection

\begin{tabular}{|l|c|c|}
\hline Variables & Number $(\mathrm{n}=24)$ & Percent \\
\hline Staph. Aureus & 8 & $33.33 \%$ \\
\hline Staph. Epidermis & 4 & $16.66 \%$ \\
\hline Staph. Pyogenes & 6 & $25 \%$ \\
\hline Strptococcus epidermis & 1 & $4.16 \%$ \\
\hline E-Coli & 3 & $12.5 \%$ \\
\hline No growth & 2 & $8.33 \%$ \\
\hline
\end{tabular}

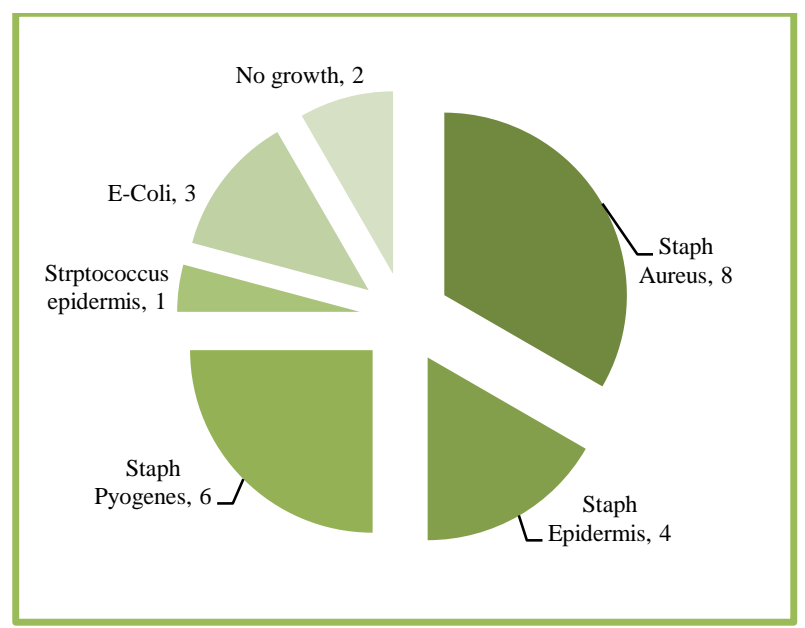

Fig. (7): Microbiological results in 24 patients with sternal wound infection.

Table (9) represents comparison of preoperative demographic and clinical characteristics in patients with sternal wound infection (SWI) versus control. Patients with SWI had significant higher differences in frequencies of obesity, diabetes mellitus, HbA1c $>7 \%$, and hepatitis $\mathrm{C}$.
Table (9):Comparison of preoperative demographic and clinical characteristics in patients with sternal wound infection (SWI) versus control

\begin{tabular}{|l|c|c|c|c|c|}
\hline \multirow{2}{*}{ Variables } & \multicolumn{2}{|c|}{$\begin{array}{c}\text { Control } \\
(\mathrm{n}=84)\end{array}$} & \multicolumn{2}{c|}{ SWI (n=24) } & \multirow{2}{*}{ P-value } \\
\cline { 2 - 5 } & No. & $\%$ & No & $\%$ & \\
\hline Age $\geq 60$ years & 4 & 4.76 & 4 & 16.66 & 0.06 \\
\hline Female gender & 46 & 54.76 & 14 & 58.33 & 0.75 \\
\hline Obesity & 25 & 29.76 & 13 & 54.16 & $0.02^{*}$ \\
\hline Current smoking & 30 & 35.71 & 8 & 33.33 & 0.82 \\
\hline Anemia & 30 & 35.71 & 10 & 41.66 & 0.59 \\
\hline Diabetes mellitus & 17 & 20.23 & 11 & 45.83 & $0.01^{*}$ \\
\hline HAlc > 7\% vascular & 11 & 13.09 & 11 & 45.83 & $0.003^{*}$ \\
\hline Hypertension & 17 & 20.23 & 9 & 37.50 & 0.08 \\
\hline $\begin{array}{l}\text { Peripheral } \\
\text { disease }\end{array}$ & 10 & 11.90 & 4 & 16.66 & 0.54 \\
\hline $\begin{array}{l}\text { Cerebrovascular } \\
\text { disease }\end{array}$ & 5 & 5.95 & 3 & 12.50 & 0.28 \\
\hline Renal dialysis cardiac & 7 & 8.33 & 5 & 20.83 & 0.08 \\
\hline $\begin{array}{l}\text { Chronic pulmonary } \\
\text { disease }\end{array}$ & 10 & 11.90 & 6 & 25 & 0.11 \\
\hline Hepatitis C & 18 & 21.42 & 10 & 41.66 & $0.04^{*}$ \\
\hline $\begin{array}{l}\text { Previous } \\
\text { surgery }\end{array}$ & & & 12.50 & 0.09 \\
\hline
\end{tabular}

*Significant difference

Table (10) represents comparison of preoperative echocardiographic characteristics in patients with sternal wound infection (SWI) versus control. Patients with SWI had significant higher differences in frequency of mitral valve disease. Other echocardiographic parameters of interest showed non-significant differences between both groups,

Table (10): Comparison of preoperative echocardiographic characteristics in patients with sternal wound infection (SWI) versus control

\begin{tabular}{|l|c|c|c|c|c|}
\hline \multirow{2}{*}{ Variables } & \multicolumn{2}{|c|}{$\begin{array}{c}\text { Control } \\
(\mathrm{n}=84)\end{array}$} & \multicolumn{2}{c|}{ SWI (n=24) } & \multirow{2}{*}{$\begin{array}{c}\text { P- } \\
\text { value }\end{array}$} \\
\cline { 2 - 5 } & No. & $\%$ & $\begin{array}{c}\text { No } \\
.\end{array}$ & $\%$ & \\
\hline Low ejection fraction & 26 & 30.95 & 10 & 41.66 & 0.32 \\
\hline Dilated left atrium & 60 & 71.42 & 20 & 83.33 & 0.24 \\
\hline $\begin{array}{l}\text { Severe pulmonary } \\
\text { hypertension }\end{array}$ & 38 & 45.23 & 16 & 66.66 & 0.06 \\
\hline Mitral valve disease & 61 & 72.61 & 23 & 95.83 & $0.01 *$ \\
\hline Aortic valve disease & 44 & 52.38 & 12 & 50 & 0.83 \\
\hline $\begin{array}{l}\text { Tricuspid } \\
\text { regurgitation }\end{array}$ & 45 & 53.57 & 17 & 70.83 & 0.13 \\
\hline
\end{tabular}

* Significant difference

Table (11) represents comparison of preoperative anti-septic measures in patients with sternal wound infection versus control. Patients with SWI had 
significant lower frequencies of preoperative day and night showers, and use of showering antiseptics. Table (11): Comparison of preoperative anti-septic measures in patients with sternal wound infection versus control

\begin{tabular}{|c|c|c|c|c|c|}
\hline \multirow{2}{*}{ Variables } & \multicolumn{2}{|c|}{ Control $(n=84)$} & \multicolumn{2}{|c|}{ SWI $(n=24)$} & \multirow{2}{*}{ P-value } \\
\hline & No. & $\%$ & No. & $\%$ & \\
\hline \multicolumn{5}{|l|}{ Hair removal: } & \multirow{3}{*}{0.71} \\
\hline Shaving & 71 & 84.52 & 21 & 87.50 & \\
\hline Clipping & 13 & 15.47 & 3 & 12.50 & \\
\hline \multicolumn{5}{|l|}{ Skin preparation: } & \multirow{3}{*}{0.16} \\
\hline Betadine and alcohol & 26 & 31 & 0 & 0 & \\
\hline Betadine or alcohol & 58 & 69 & 24 & 100 & \\
\hline \multicolumn{5}{|l|}{ Shower: } & \multirow{3}{*}{$0.001 *$} \\
\hline Night & 33 & 39.28 & 19 & 79.16 & \\
\hline Day and night & 51 & 60.71 & 5 & 20.83 & \\
\hline \multicolumn{5}{|l|}{ Showering antiseptic } & \multirow{3}{*}{$\begin{array}{c}0.0001 \\
*\end{array}$} \\
\hline Yes & 50 & 59.53 & 4 & 16.66 & \\
\hline No & 34 & 40.47 & 20 & 83.33 & \\
\hline
\end{tabular}

* Significant difference

Table (12) represents comparison of operative and postoperative outcomes in patients with sternal wound infection versus control. Patients with SWI had significant higher frequencies of prolonged bypass time $>120 \mathrm{~min}$, use of inotropes, blood loss $>500 \mathrm{ml}$ in ICU and prolonged ventilation > 12 hours.

Table (12): Comparison of operative and postoperative outcomes in patients with sternal wound infection versus control

\begin{tabular}{|l|c|c|c|c|c|}
\hline \multirow{2}{*}{ Variables } & \multicolumn{2}{|c|}{$\begin{array}{c}\text { Control } \\
(\mathrm{n}=84)\end{array}$} & \multicolumn{2}{c|}{ SWI (n=24) } & \multirow{2}{*}{$\begin{array}{c}\text { P- } \\
\text { value }\end{array}$} \\
\cline { 2 - 5 } & No. & $\%$ & $\begin{array}{c}\text { No } \\
.\end{array}$ & $\%$ & \\
\hline Bypass time > 120 min & 6 & 7.14 & 6 & 25 & $0.01 *$ \\
\hline Cross clamp time > 60 min & 40 & 47.61 & 16 & 66.66 & 0.10 \\
\hline Blood loss > 500 ml & 14 & 16.66 & 10 & 41.66 & $\begin{array}{c}0.009 \\
*\end{array}$ \\
\hline Re-exploration for bleeding & 3 & 3.57 & 3 & 12.50 & 0.09 \\
\hline Ventilation time > 12h & 5 & 5.95 & 5 & 20.83 & $0.02 *$ \\
\hline $\begin{array}{l}\text { Removal of drains after } 3 \\
\text { days }\end{array}$ & 49 & 58.33 & 15 & 62.50 & 0.71 \\
\hline
\end{tabular}

* Significant difference

Table (13) represents significant perioperative risk factors associated for sternal wound infection using univariate regression analysis. Calculating Odds-
Ratio (OR), 95\% confidence interval (CI) and pvalue obtained using binary logistic regression.

Perioperative risk factors of significant odds ratio for sternal were: obesity, diabetes mellitus, elevated $\mathrm{HbA} 1 \mathrm{c}>7 \%$, history of hepatitis $\mathrm{C}$, surgery for mitral valve disease, preoperative use of only night shower, no use of showering antiseptic, prolonged bypass time $>120 \mathrm{~min}$, blood loss $>500 \mathrm{ml}$ in ICU, and prolonged mechanical ventilation $>12$ hours.

Table (13): Significant perioperative risk factors associated for sternal wound infection using univariate regression analysis

\begin{tabular}{|l|l|l|l|l|}
\hline \multirow{2}{*}{ Variables } & \multirow{3}{*}{ OR } & \multicolumn{2}{|l|}{$\begin{array}{l}\text { Interval } \\
\text { Intenfidence }\end{array}$} & \multirow{2}{*}{ P-value } \\
\cline { 3 - 4 } & & $\begin{array}{l}\text { Lower } \\
\text { bound }\end{array}$ & $\begin{array}{l}\text { Upper } \\
\text { bound }\end{array}$ & \\
\hline Obesity & 2.78 & 1.10 & 7 & $0.03^{*}$ \\
\hline Diabetes mellitus & 3.33 & 1.29 & 8.58 & $0.01^{*}$ \\
\hline HbA1c > 7\% & 4.83 & 1.70 & 13.69 & $0.003^{*}$ \\
\hline Hepatitis C & 2.61 & 1.01 & 6.75 & $0.04^{*}$ \\
\hline Mitral valve disease & 8.67 & 1.55 & 48.31 & $0.01^{*}$ \\
\hline Only night shower & 0.17 & 0.06 & 0.48 & $0.001^{*}$ \\
\hline $\begin{array}{l}\text { No showering } \\
\text { antiseptic }\end{array}$ & 0.13 & 0.04 & 0.41 & $0.0001^{*}$ \\
\hline $\begin{array}{l}\text { Bypass time >120 } \\
\text { min }\end{array}$ & 4.33 & 1.30 & 14.37 & $0.01^{*}$ \\
\hline Blood loss > 500 ml & 3.57 & 1.34 & 9.46 & $0.009^{*}$ \\
\hline Ventilation > 12h & 4.15 & 1.15 & 14.97 & $0.02^{*}$ \\
\hline
\end{tabular}

* Significant risk factor on univariate analysis

Table (14) represents results of multivariate regression analysis of the univariate significant perioperative risk factors associated for sternal wound infection. The significant predictors were obesity (OR: 10.2, 95\% CI: 1.2-86, p = 0.03), uncontrolled hyperglycemia with HA1c $>7 \%$ (OR: 20.4, 95\% CI: 1.06-393, $\mathrm{p}=0.04)$, and disuse of preoperative showering antiseptic (OR: 0.07, 95\% CI: 0.009-0.58, $\mathrm{p}=0.01$ ). 
Table (14): Multivariate regression analysis of univariate significant perioperative risk factors associated for sternal wound infection

\begin{tabular}{|l|c|c|c|c|}
\hline \multirow{2}{*}{ Variables } & \multirow{2}{*}{ OR } & \multicolumn{2}{|c|}{$\begin{array}{c}\text { 95\% Confidence } \\
\text { Interval }\end{array}$} & \multirow{2}{*}{$\begin{array}{c}\text { P- } \\
\text { value }\end{array}$} \\
\cline { 3 - 4 } & & $\begin{array}{c}\text { Lower } \\
\text { bound }\end{array}$ & $\begin{array}{c}\text { Upper } \\
\text { bound }\end{array}$ & \\
\hline Obesity & 10.2 & 1.2 & 86 & $0.03 *$ \\
\hline Diabetes mellitus & 0.19 & 0.01 & 3.57 & 0.27 \\
\hline HA1c > 7\% & 20.4 & 1.06 & 393 & $0.04 *$ \\
\hline Hepatitis C & 1.29 & 0.27 & 6 & 0.74 \\
\hline Mitral valve disease & 4.66 & 0.30 & 71.9 & 0.27 \\
\hline Only night shower & 0.47 & 0.08 & 2.59 & 0.38 \\
\hline $\begin{array}{l}\text { No showering } \\
\text { antiseptic }\end{array}$ & 0.07 & 0.009 & 0.58 & $0.01 *$ \\
\hline Bypass time >120 min & 1.67 & 0.18 & 15.5 & 0.65 \\
\hline Blood loss > 500 ml & 0.29 & 0.02 & 4.32 & 0.37 \\
\hline Ventilation > $12 \mathrm{~h}$ & 21.9 & 0.85 & 565 & 0.06 \\
\hline
\end{tabular}

*Significant predictor

\section{Discussion}

SSI can double the length of time a patient stays in hospital and thereby increase the costs of health care. Additional costs attributable to SSI have been reported depending on the type of surgery and the severity of the infection (Coello et al., 2005).

Sternal wound infection leads to prolonged hospital stay and is responsible for in-hospital mortality rates of 8-28\% (Baillot et al., 2010; Vos et al., 2014).

As a type of SSIs, sternal wound infection (SWI) is a significant complication after median sternotomy for cardiac surgery (Kirmani et al., 2013).

The incidence of SWI has barely declined over the last decades. This may be explained by the fact that cardiac surgery is more frequently performed in patients with higher risk of infection, despite improvement in preventive measures (Dubert et al., 2015).

Therefore, prevention of SWI is better than cure, thus determining and management of the factors associated with sternal wound infection is an important issue. With meticulous attention to perioperative risk factors sternal wound complications can be reduced significantly (Dogra et al., 2005).

Searching for cheaper and efficient methods of prevention of sternal wound infection, a rigorous attention to the details of preoperative, intraoperative, and postoperative management could contribute to keep SWIs at a minimal rate (Cotogni et al., 2015).

Numerous studies have identified patient-related and procedure-related risk factors including: age, female gender, obesity, diabetes mellitus, chronic obstructive pulmonary disease (COPD), peripheral arterial occlusive disease, duration of the operation, reoperation for bleeding, sternal rewiring, extensive electrocautery, and shaving with razors, as independent predictors for development of sternal wound infections after cardiac operations (Gummert et al., 2002; Braxton et al., 2004; Lemaignen et al., 2015).

Risk Factors for sternal wound infection after open cardiac surgery vary according to type of operation. Little number of studies focused on risk factors that may depend on the type of surgical procedure (Borger et al., 1998; Meszaros et al., 2016).

Therefore, we agree with the hypothesis that occurrence of SWI is due to several factors and its prevention relies on the control of risk factors related to patients, to the procedure, and to hospital environment, during the pre-, intra-, and postoperative periods (Lepelletier et al., 2013).

In this scope, the aim of the present study was to investigate the perioperative predictors of sternal wound infection in a consequent cohort of patients undergoing cardiac surgery for management of different types of heart valve diseases.

Antimicrobial prophylaxis

In the present study, certain measures to prevent SSIs were applied as a rule at our institution including antimicrobial prophylaxis. Second- or third-generation Cephalosporin were routinely given to all patients about 60 min before surgery.

The prophylactic antibiotic therapy is one of the most important tools in the prevention of SWI. The advantages of proper antimicrobial prophylaxis in patients undergoing cardiac surgery have been clearly demonstrated (Spellberg et al., 2011). However, the choice of antibiotic, the dose, the 
duration, the adequate levels in serum and tissue, and the timing of antimicrobial prophylaxis are still controversial.

The Society of Thoracic Surgeons Practice Guidelines on antimicrobial prophylaxis in cardiac surgery recommended that a cephalosporin should be given within $60 \mathrm{~min}$ from the skin incision and be continued for 24-72 hours (Edwards et al., 2006; Engelman et al., 2007).

Evidence supports second- or third-generation cephalosporin for cardiac surgery prophylaxis and points at a possible advantage of prophylaxis prolongation up to $48 \mathrm{~h}$ post-operatively (Lador et al., 2012).

A meta-analysis comparing cephalosporin with glycopeptides as antimicrobial prophylaxis regimens found a higher frequency of postoperative SSIs and a trend toward an increased risk of Grampositive SSI in the glycopeptide group but a lower frequency of SSIs caused by resistant gram-positive pathogens (Bolon et al., 2004).

The relationship between timing of prophylactic antimicrobial administration and risk of infection is an additional field of debate. The 2011 American College of Cardiology/American Heart Association guidelines for cardiac surgery recommend that "Antibiotic prophylaxis should be initiated 30 to 60 min before surgery" (Hillis et al., 2011).

Key studies have demonstrated that antimicrobial prophylaxis administered too late or too early reduces the efficacy of the antimicrobial prophylaxis and increases the risk of infection (Cotogni et al., 2013); conversely, other reports do not clearly demonstrated the superiority of the 1hour window (Hawn et al., 2013).

\section{Classification and types of SWI}

In the present study, we classified sternal wound infections into superficial and deep. It is important to distinguish deep sternal wound infections (DSWIs) from superficial sternal wound infections (SSWIs). This classification is of special importance as SSWIs are often completely eradicated with intravenous antibiotics and local wound care if necessary without long-term sequelae (Singh et al.,
2011).

In the present study, we defined DSWI in accordance to the classification scheme introduced by El Oakley and Wright which is based on the time at which the patient presents with mediastinitis relative to the initial surgical procedure. El Oakley description accounts for relevant risk factors underlying the clinical scenario and whether or not previous attempts to treat the sternal wound infection have been made and failed. Therefore, five distinct categories of infection are described, each with important treatment implications. For example, the subtypes I and II appear to respond well to primary sternal closure with mediastinal irrigation, while subtypes III-V appear to require more aggressive sternal debridement and repair techniques. This descriptive classification scheme provides more specific insight into the pathologic involvement of the sternal tissues and the clinical consequences and course (El Oakley and Wright 1996; Schulman and Subramanian, 2004).

In contrast, the criteria of medistinitis provided by the U.S. Centers for Disease Control and Prevention (CDC) which include: (1) an organism isolated from culture of mediastinal tissue or fluid; (2) evidence of mediastinitis seen during operation; or (3) presence of either chest pain, sternal instability, or fever $(>380 C)$, and either purulent drainage from the mediastinum, isolation of an organism present in a blood culture, or culture of the mediastinal area (Mangram et al., 1999).

The definition provided by CDC is somewhat deficient since either superficial infections, confined to the soft tissues, or deep infections, involving the bone and/or retrosternal space could produce bacteremia and clinical signs of severe infection (El Oakley and Wright, 1996).

Incidence of SWI

In the present study, the incidence of superficial sternal wound infection was $19.44 \%$ and the incidence of deep sternal wound infection was $2.77 \%$.

These incidences are in agreement with the contemporary data in literature. Despite many 
advances in prevention, deep sternal wound infection remains a significant postoperative complication and its incidence ranges from $0.5 \%$ to 6.8\% (Filsoufi et al., 2009; Bryan and Yarbrough, 2013).

The incidence of superficial sternal infection exceeds that of deep sternal infection by as many as three times (Ridderstolpe et al., 2001), and it had been stated that as many as $70 \%$ of patients with post-sternotomy infection will have superficial tissue involvement (Francel, 2004).

Risk factors

In the present study, the significant predictors of SWI after valve surgery were obesity (OR: 10.2, $95 \%$ CI: $1.2-86, \mathrm{p}=0.03$ ), poor glycemic control with elevated HA1c > 7\% (OR: 20.4, 95\% CI: $1.06-$ $393, \mathrm{p}=0.04)$, and disuse of antiseptic on preoperative bathing or showering (OR: $0.07,95 \%$ CI: 0.009-0.58, $\mathrm{p}=0.01$ ).

These findings are consistent with other studies in literature. Several different patient-related factors have been repeatedly implicated in the development of SWI, most consistently obesity and diabetes mellitus, more precisely poor glycemic control (Prabhakar et al., 2002; Eklund et al., 2006; Lemaignen et al., 2015; Meszaros et al., 2016).

However, most of the studies in literature aim to estimate risk factors of SWI focus on SWI after coronary artery surgery, and did not differentiate risk factors in relation to other types of cardiac surgery.

The current study by Meszaros et al. (2016) found that risk factors for sternal wound infections after cardiac operations vary with the type of surgical procedure. In valve patients, revision for bleeding and diabetes mellitus were independent predictors for sternal infection. For combined valve and CABG operations, revision for bleeding and duration of operation exceeding 300 minutes were independent predictors for sternal infection.

Other studies identified predictors of SWI after cardiac surgery, but with no special concern for valve surgery. In the study by Olsen et al. (2002), obese diabetic patients had an increased risk of deep chest infections and the independent risk factors for superficial surgical-site infections included obesity, diabetes in persons 65 years of age or older, and current smoking. Use of antiplatelet drugs was associated with a lower risk of superficial infections.

The study Prabhakar et al. (2002) showed that the risk for major perioperative complications, particularly deep sternal wound infection increased for extremely obese patients.

In the large study by Fowler et al. (2005), the risk factors associated with major infection after cardiac surgery included body mass index (BMI) $>40$ $\mathrm{kg} / \mathrm{m} 2$; hemodialysis, cardiogenic shock, age $>85$ years, immunosuppressive treatment and diabetes mellitus.

In the study by Cayci et al. (2008) preoperative predictors of DSWI were BMI $>30 \mathrm{~kg} / \mathrm{m} 2$, diabetes mellitus, urgent operation, smoking history within past year, smoking history within past 2 weeks, and a history of stroke.

The independent predictors of all sternal wound complications in the study by Risnes et al. (2010) included: obesity (BMI > $30 \mathrm{~kg} / \mathrm{m} 2$ ), insulindependent diabetes, smoking, and prolonged ventilator support.

The mechanism by which obesity increases the risk of SSI is likely to be multifactorial. Obese surgical patients have been shown to have reduced subcutaneous tissue oxygenation and to require a greater fraction of inspired oxygen to achieve the same arterial oxygen tension as normal-weight patients, thus predisposing them to SSI (Kabon et al., 2004).

Wound hypoxia impairs healing by a number of potential mechanisms; healing wounds have high metabolic demands, and insufficient oxygen will slow the healing process. Immune cells also have high oxygen demands, requiring oxygen for the formation of microbicidal reactive oxygen species (Sen, 2009).

In addition to poor tissue oxygenation, adequate tissue levels of prophylactic antibiotics may be harder to achieve in obese patient. Antimicrobials 
show different pharmacokinetics when administered to obese patients, with both hydrophilic and hydrophobic compounds generally having a higher volume of distribution, requiring a higher dose to reach the same plasma drug concentrations as for non-obese patients. Hepatic clearance may also be increased in obese patients. Therefore, obese patients may need to be dosed differently from nonobese patients (Falagas and Karageorgopoulos, 2010; Chopra et al., 2010).

The present study identified poor glycemic control as determined by elevated $\mathrm{HbAlc}$, as a predictor of SWI after valve surgery. Glycosylated hemoglobin (hemoglobin A1c, HbA1c) is a sensitive and reliable biomarker that provides a measure of glucose control over the prior months (Kramer et al., 2008).

The American Diabetes Association (ADA) suggests an HbA1c goal of $7 \%$ or lower to reduce the long-term complications of DM (American Diabetes Association, 2011).

Previous studies suggest a link between poor glycemic control and surgical site infection rates (El Oakley et al., 1997; Zerr et al., 1997; Furnary et al., 2004; Fowler et al., 2005).

This finding is supported by the current evidence which recommend optimizing glycemic control in patients with increased $\mathrm{HbAlc}$ levels and serum glucose levels $>200 \mathrm{mg} / \mathrm{dL}$ before any cardiac surgery procedure (Lazar et al., 2016).

Therefore, efforts should be made to optimize glucose control before surgery because this has been associated with an increased incidence of SWI (Zerr et al., 1997).

In patients who require urgent or emergent surgery in whom serum glucose levels are persistently $>180$ $\mathrm{mg} / \mathrm{dL}$, intravenous insulin infusions are the most effective method to rapidly achieve glycemic control. These infusions should be continued in the intra- and postoperative periods if necessary to maintain serum glucose levels $<180 \mathrm{mg} / \mathrm{dL}$ (Lazar et al., 2004).

The results of the present study revealed an association of disuse of preoperative antiseptic bathing or showering with SWI. In other studies, preoperative bathing or showering using $4 \%$ Chlorhexidine gluconate has been shown to significantly reduce microbial counts on the skin (Byrne and Cuschieri, 1992).

A study comparing the efficacy of pre-operative showering or bathing with chlorhexidine to other wash products in reducing surgical site infection in general surgery, vascular surgery and urology patients failed to demonstrate a benefit (Webster and Osborne, 2007).

A theoretical advantage of using chlorhexidine is that in addition to reducing staphylococcus aureus on the skin it also reduces coagulase negative staphylococci and other micro-organism such as gram negative bacteria (Mauermann and Sampathkumar, 2008).

In contrast, the findings regarding the role of presurgical bathing with antinseptic to reduce SSI are still controversial. The study by Kamel et al. (2012) showed no difference in the postoperative infection rates between patients who showered with chlorhexidine, povidone-iodine, soap and water, or placebo.

The study by Kuhme and Colleagues isolated coagulase-negative staphylococci and Propionibacterium acnes from the subcutaneous tissue in $89 \%$ of patients undergoing cardiac surgery and from the skin surrounding the wound in $98 \%$ of those patients who washed with a preoperative chlorhexidine scrub. The authors concluded that skin preparation with chlorhexidine alone cannot prevent skin flora organisms from contaminating the wound and surrounding tissue during cardiac surgical procedures, but will reduce bacterial counts (Kuhme et al., 2007).

\section{Clinical presentation}

In the present study, clinical presentation of SWI included wound discharge in $67 \%$ and wound discharge with fever in $33 \%$.

This result is consistent with the reported findings in literature showing that the most common clinical presentation of SWI includes: sternal wound drainage, sternal instability, fever, and malaise. In many cases, a high index of suspicion is required to 
establish the diagnosis, especially when classic signs and symptoms are absent.

It is often difficult to distinguish on physical examination the difference between deep sternal wound infection (infection present beneath the sternum) and superficial sternal infection. Therefore, early wound opening and inspection with appropriate sampling of tissue for bacteriologic assessment is strongly encouraged when sufficient clinical suspicion exists (Atkins and Wolfe, 2012).

Microbiology

In the present study, the causative organisms of SWI were staph. aureus in $33.33 \%$, followed by staph pyogenes in $25 \%$, staph. epidermis in $16.66 \%$, streptococcus epidermis in $4.16 \%$ and E-coli in $12.5 \%$.

It appears that the majority of bacteria isolated from sternal wound infections continue to be gram positive organisms (Soderquist, 2007; Chan et al., 2016).

In the Western countries, Staphylococcus aureus seems to be the most commonly isolated organism from sternal wounds (Sharma et al., 2004; Filsoufi et al., 2007).

Similarly, the current Egyptian studies reported gram positive cocci, Staphylococcus aureus as the most commonly isolated organism from SWI (Mansour and Mellitt, 2009; Allam and Hassanein, 2013).

The Staphylococci are metabolically well equipped to cause infection. They have numerous virulence factors including adhesion proteins, exotoxins, and enzymes that interfere with the native immune response. They also contain enzymes that cause local tissue necrosis and allow the infection to spread (Soderquist, 2007).

Surgical site infections after clean procedures primarily result from micro-organisms introduced at the time of surgery. Surgical wounds may become contaminated with Staphylococci either by exogenous exposure from healthcare workers or endogenously from the patient's own nasal flora. It has been shown that Staphylococcus aureus may be transferred between patients via the hands of healthcare workers (Eklund et al., 2002).

Despite the potential for transmission from healthcare workers, it appears that the endogenous route of S. aureus is more common. Nasal carriage of S. aureus has been a known risk factor for the development of SSIs for decades. The modern technology show that $\mathrm{S}$. aureus isolated from SSIs is often genetically identical to that which is isolated from the patient's nasal flora (Sollid et al., 2014).

Jakob and colleagues studied 376 patients undergoing cardiac surgery. Cultures were taken from the nares of all patients pre- and postoperatively and from the wounds during surgery and during dressing changes. Nasal cultures were taken from all nursing and medical staff as well. There were 25 sternal wound infections (16 superficial and 9 deep) and S. aureus was isolated from 12 of the infected wounds. In all but one of these 12 patients, the $\mathrm{S}$. aureus was genetically identical to that of the patient's nasal flora (Jakob et al., 2000).

While hand hygiene by healthcare workers will continue to be an important step in preventing transmission of organisms, current data suggests that the patient's endogenous nasal and skin flora may pose a more significant risk for the development of DSWI. This is one potential target for a strategy to decrease the incidence of SSIs after cardiac surgery (Jakob et al., 2000; Mauermann and Sampathkumar, 2008).

Conflict of interest: All authors declare that they have no conflict of interest.

\section{References}

1. Gummert JF, Barten MJ, Hans C, et al. Mediastinitis and cardiac surgery-an updated risk factor analysis in 10.373 consecutive adult patients. Thorac Cardiovasc Surg 2002; 50: 87-91.

2. Braxton HJ, Marrin CA, McGrath PD, et al. 10-year follow-upof patients with and without mediastinitis. Semin Thorac Cardiovasc Surg 2004; 16: 70-6. 
3. Horan TC, Andrus $\mathrm{M}$ and Dudeck MA. CDC/NHSN surveillance definition of health care-associated infection and criteria for specific types of infections in the acute care setting. Am J Infect Control 2008;36: 30932.

4. Mangram AJ, Horan TC, Pearson ML, et al. Guideline for Prevention of Surgical Site Infection, 1999. Centers for Disease Control and Prevention (CDC) Hospital Infection Control Practices Advisory Committee. Am J Infect Control. 1999; 27(2):97-132.

5. Fowler VG, O'Brien SM, Muhlbaier LH, et al. Clinical predictors of major infections after cardiac surgery. Circulation 2005; 112(1): I-358-65.

6. Cayci C, Russo M, Cheema FH, et al. Risk analysis of deep sternal wound infections and their impact on long-term survival: a propensity analysis. Ann Plast Surg 2008; 61: 294-301.

7. Fakih MG, Sharma M, Khatib $R$, et al. Increase in the rate of sternal surgical site infection after coronary artery bypass graft: a marker of higher severity of illness. Infect Control Hosp Epidemiol 2007; 28: 655-660.

8. Steingrimsson $\mathrm{S}$, Gottfredsson $\mathrm{M}$, Gudmundsdottir I, et al, Negative-pressure wound therapy for deep sternal wound infections reduces the rate of surgical interventions for early re-infections. Interact Cardiovasc Thorac Surg 2012; 15: 406-410. 